\title{
Treatment of Chronic Endometritis With the Recruitment of Local Homeostasis and Reproductive Function
}

\author{
Tsallagova L.V. ${ }^{1,2,}$ Kabulova I.V., ${ }^{1,2}$ Maisuradze L.V. ${ }^{1,2}$ Zoloyeva I.A. ${ }^{3}$ \\ Popova L.S. ${ }^{1,2}$ Dzagoeva Z.L. ${ }^{2}$ Alborov D.K. ${ }^{4}$ Gabueva Ya.O. ${ }^{2}$ \\ ${ }^{1}$ Department of Pathology of Mother and Child. Institute of biomedical research - the branch of the Vladikavkaz \\ Scientific Center of the Russian Academy of Sciences, Vladikavkaz, Russia. \\ ${ }^{2}$ Department of Obstetrics and Gynecology No. 1, the North Ossetian State Medical Academy of the Ministry of Health of \\ the Russian Federation, Vladikavkaz, Russia \\ ${ }^{3}$ Ardonskaya Central District Hospital MZ RSO-Alaniya, the city of Ardon, Russia \\ ${ }^{4}$ State Medical Institution "Maternity hospital No. 2" MZ RSO-Alaniya, Vladikavkaz, Russia \\ *Corresponding author. Email: akusherstvo_1@mail.ru
}

\begin{abstract}
The treatment of infertility associated with chronic endometritis has become the most widely discussed aspect of this complex problem in recent years. The studies were performed based on the examination and treatment of 80 patients of reproductive age with a morphologically verified diagnosis of chronic endometritis. The treatment included antibacterial, anti-inflammatory, antimicrobial, metabolic, and hormonal medications. 10 apparently healthy women of reproductive age were selected for the comparison group. After the treatment, echographic and Doppler's parameters showed the improved endometrial structure and positive hemodynamic changes in all patients of the experimental group. In the experimental group, the cases of pregnancy occurred in $46 \%$ of patients with chronic endometritis and reproductive disorders, while in the control group - in 17 $\%$, and this indicates the high effectiveness of the developed treatment regimen.
\end{abstract}

Keywords: chronic endometritis, reproductive function, intrauterine therapy, pelvic organs, infertility

\section{INTRODUCTION}

According to various authors, the frequency of chronic endometritis varies from 3 to $72 \%$ among all gynaecological diseases, while the increase in this indicator is associated with an increase in the frequency of chronic pelvic inflammatory diseases [1-3].

The interest of most researchers in chronic endometritis, as a separate nosology from the total number of chronic inflammatory diseases of the pelvic organs, has increased significantly in recent years, since there are many problems associated with solving the issues of etiopathogenesis, diagnosis and development of modern methods of treatment of this pathology. In cases of chronic endometritis, the disorders relate to both menstrual and reproductive functions, which ultimately leads to the development of infertility, miscarriage, unsuccessful IVF attempts, endometrial hyperplasia, and sexual problems. In modern literature, it is noted that chronic endometritis is a medical and social problem, taking into account the active reproductive age of most female patients. Nowadays, chronic endometritis is a clinical and morphological syndrome in which multiple secondary morphological and functional changes occur due to persistent endometrial damage caused by infectious factors that violate the cyclic biotransformation and receptivity of the uterine body mucosa [4-6].

According to different authors in cases of chronic endometritis noted the close relationship between inflammatory and immunohistopathology changes. In modern conditions, the role of environmental imbalance is high, the pollution of which further reduces the immune status of female patients, and the widespread uncontrolled usage of antibiotics in inflammatory processes of the genitals exacerbates these disorders, simultaneously contributing to the growth of resistant strains of microorganisms. The normal microflora of the body is suppressed, microbial factors that lead to chronic endometritis evolve, associations of microorganisms in the endometrium become predominant and, ultimately, this reduces the effectiveness of treatment for women with chronic endometritis [7, 8]. An important process in the formation of receptivity is angiogenesis - the process of formation of new blood vessels, which ensures the functional preparation of the endometrium and its readiness for implantation, since the endometrium is a key structure in the providing implantation, which is a process of complex integration and interchange between the gestational sac and endometrial cells. The duration and often oligosymptomatic of persistent infectious agents in the endometrial tissue can lead to its evident structural 
changes, causing dysfunction of the processes of proliferation and cyclic transformation of the endometrium, disrupting physiological implantation and placentation, resulting in the formation of an inadequate pathological response to the onset of pregnancy.

Much remains unsolved in the issues of etiopathogenesis, diagnosis and treatment of chronic endometritis. There are still discussions about the mechanisms of reproductive disorders in cases of chronic endometritis and the peculiarities of the caused changes. Research by modern scientists is also aimed at studying the molecular and biological processes that affect the receptivity of the endometrium.

In these conditions, new methods of therapy for chronic endometritis are of particular interest, which will combine both high efficiencies concerning the most common infectious agents, and reduce the drug load on the body, while not violating the normal microcenosis of the vagina, and, consequently, allow restoring the reproductive function of female patients.

The study aims to evaluate the effectiveness of complex therapy of chronic endometritis in pelvic homeostasis disorders using local etiotropic administration of drugs into the uterus and pelvis.

\section{METHODS AND MATERIALS}

A complete clinical and laboratory physical examination was performed in 80 patients with chronic endometritis and infertility. The age of the female patients ranged from 21 to 42 years. Typical complaints during treatment were pain and discomfort in the lower abdomen, the presence of secretions from the genital tract, sometimes accompanied by an unpleasant smell, irregular menstruation, the absence of a desired pregnancy with regular sexual life without the use of contraception.

When selecting patients, the cases of hypoplastic, hyperplastic processes and endometrial polyposis were excluded. The patients were divided into two groups by random selection. In the first control group, including 30 patients with chronic endometritis, traditional therapy was performed. In the second, experimental group, including 50 patients, in addition to traditional therapy, drugs were administered to the uterus and pelvis using the author's method. The comparison group involved 10 apparently healthy women.

To detect infectious agents and diagnose chronic endometritis, the following studies were conducted: smears to diagnose vaginal flora, bacteriological cultures to detect microflora and sensitivity to antibiotics, polymerase chain reaction to identify the genome of the most common possible pathogens of chronic endometritis (gonococci, Mycoplasma, Ureaplasma, Chlamydia, Trichomonas, Gardnerella, viruses), cytological studies of the cervix, echographic studies with Doppler velocimetry of blood flow in the uterine vessels, hysteroscopic methods with the separate diagnostic scraping of the uterine cavity, histological examination of endometrial scrapings in the $1^{\text {st }}$ phase of the menstrual cycle, hormonal research methods.
All patients underwent pelvic ultrasound examination on the $5^{\text {th }}-7^{\text {th }}$ and $17^{\text {th }}-21^{\text {st }}$ days of the menstrual cycle using the Toshiba Aplio300 device. We used vaginal and abdominal sensors with frequencies of $6.0-5.0 \mathrm{MHz}$, respectively, to detect echo signs of chronic endometritis and provide Spectral Doppler evaluation of uterine vessels. A Doppler study of blood flow was performed in the uterine arteries. Immunohistochemical examination of the endometrium was performed by a two-stage avidin-biotin complex method with the unblinding of the antigen on paraffin sections. The method of immunohistochemistry has $100 \%$ sensitivity to plasmocytes and more fully allows getting a visualization of CD138 in the form of brown staining of cell membranes and cytoplasm. Determination of markers of endometrial immunocompetent cells by the immunohistochemical method was performed by studying endometrial lymphocytes expressing markers of natural killer cells CD56+ and CD16+ and lymphocytes expressing the activation marker HLA DR+. The expression of estrogen and progesterone receptors was studied. The analysis of the results for ER- $\alpha$ and PR was performed taking into account the number of coloured cells and the intensity of colour in the surface epithelium, glands and endometrial stroma, using the histological counting method Histoscore. The degree of expression of ER and PR was assessed as 0-10\% - lack of expression, 11-100\% - weak expression, 101-200\% - moderate expression, 201-300\% - severe expression.

Hysteroscopy was performed to visualize the uterine cavity and diagnose chronic endometritis in the proliferative phase of the menstrual cycle and was combined with a targeted endometrial biopsy or traditional diagnostic scraping of the uterine cavity with the mandatory morphological examination of materials.

All patients with diagnosed chronic endometritis were treated for pelvic inflammatory diseases and their reproductive health was also evaluated. In the control group of patients, etiotropic therapy was prescribed, including antibiotics, antiseptics, anti-inflammatory, desensitizing, hormonal and general revitalising medicines. Patients of the experimental group were treated locally for 10 days, starting from the 3rd-4th day after the start of traditional therapy, while antibacterial, immunomodulatory and proteolytic drugs were injected into the uterine and pelvic cavities. The following treatment method was developed: administering of Miramistin $(5 \mathrm{ml})$ into the uterine cavity, after 5 minutes Cefazoline or Ceftriaxone, after 5 minutes - Derinate $(5 \mathrm{ml})$ alternating the next day with Longidase $(3,000$ units) using a Foley catheter into the uterine cavity, followed by drugs entering the pelvic cavity.

Female patients at the second stage of treatment received hormone therapy with progesterone drugs (Duphaston, Utrozhestan) for 3 months.

Statistical processing of the research results was performed using the Excel spreadsheet package. We used the t-criterion, which is applied for normally distributed variables when the dispersion of observations in groups is not too different. The normality of the distribution was 
checked with the aid of a visual study using a histogram and the Kolmogorov-Smirnov and Lilliefors normality criteria in the STATISTICA-13 program. The equality of dispersion in the two groups was checked using the $F$ criterion.

\section{RESULTS}

After completing all stages of therapy, the patients of the experimental group had no complaints. In the control group, after regular menstruation, $63 \%$ of patients experienced a return of previously disturbing complaints.

In patients with chronic endometritis and reproductive disorders, microbiological studies revealed a predominance of pathological types of vaginal microbiocenosis (bacterial vaginosis was detected in $45 \%$ of patients, non-specific vaginitis - in $21 \%$, vaginal candidiasis - in $28 \%$ ). Bacterial infections of the vagina were represented by Ureaplasma in $14 \%$ of cases, genital Mycoplasma in $10 \%$, Chlamydia in $9 \%$, Trichomonas in $8 \%$. Herpes simplex virus $(43 \%)$ and Cytomegalovirus (38 \%) predominated among the pathogens of viral infections. The most frequent associations in the examined patients were combinations of gram-negative flora, ureaplasmas, fungi, trichomonads, chlamydia with herpesvirus infection and cytomegalovirus. Bacterial vaginosis as a cause of chronic endometritis and tuboperitoneal infertility deserves to be highlighted. In our studies, bacterial vaginosis was diagnosed based on a decrease in lactobacilli in the vagina, detection of Gardnerella by smear microscopy, an alkaline environment in the vagina, and a positive $\mathrm{KOH}$ test. The conducted cultural study revealed that bacterial vaginosis has a high spectrum of non-spore-forming anaerobic microorganisms, both obligate (there were bacteroids, fusobacteria, peptostreptococci and peptococci) and facultative (coagulase-negative staphylococci).

Recovery and normalization of laboratory indicators of infectious and inflammatory conditions of the vagina were observed after treatment in $90 \%$ of the experimental group and $67 \%$ of the control group. Pathological types of vaginal microbiocenosis after treatment were determined in $17 \%$ of cases in the control group undergoing traditional treatment. In the experimental group of patients, the normalization of vaginal and cervical microbiocenosis consisted of the complete disappearance of Mycoplasmas, Ureaplasmas, Chlamydia, Gardnerella and Trichomonas under the influence of complex therapy with local intrauterine and vaginal procedures.

The clinical signs of pelvic inflammatory disease returned in all control group patients with chronic endometritis. This again confirms that in chronic endometritis, the persistence of a damaging agent in the uterine cavity is closely related to the state of vaginal microbiocenosis and requires longer-term combined systemic and local therapy.

The analysis of ultrasound and Doppler parameters of uterine vascular hemodynamics was performed in all the examined patients, as well as in 10 apparently healthy women as a comparison. In the dynamics of ultrasound examination, the most positive changes were observed in patients of the experimental group, there was no expanding of the uterine cavity, improving the endometrial echostructure and its uniformity, increasing in the thickness of the endometrium with an initial thinning of less than $7 \mathrm{~mm}$, as well as decreasing in the size and echostructure of the ovaries.

According to the literature, an important aspect that determines the implantation potential of the endometrium is hemocirculation in the uterine vascular system, measured by Doppler velocimetry. In patients with chronic endometritis, the study of hemodynamics revealed deep disorders of the vascular system of the uterine arteries. The study of dopplerograms of the uterine arteries revealed a marked increase in the pulsation index, a decrease in blood flow rates and an increase in the systolic-diastolic ratio. After treatment, in the experimental group of examined patients, when analyzing the results of the evaluation of blood flow in the uterine arteries, a positive dynamics was noted, which indicated an improvement in endometrial vascularization. A comparative analysis of the obtained data showed a significant decrease in vascular resistance, as well as an increase in blood flow rates in the uterine arteries in patients of the experimental group $(\mathrm{p}<0.01)$ who received stage-based combination therapy. Patients who underwent only traditional treatment had less marked dynamics of these parameters $(p<0.05)$. The observed positive changes in dopplerometry parameters indicated a significant improvement in the hemodynamics of uterine vessels in the examined patients of the experimental group when combined therapy and local administration of drugs into the uterine cavity and pelvic organs were used in patients with chronic endometritis.

The data obtained in the second phase of the menstrual cycle for quantitative evaluation of the expression of immunohistochemical markers of inflammation CD 16, CD 56 and HLA-DR in the endometrium were compared with indicators of systemic immunity. In $80 \%$ of patients in the control group and $82 \%$ of the experimental group, the high expression of CD 56 and HLA-DR -positive cells was registered in the endometrium, and this confirms the verified chronic endometritis in these cases. In the examined clinical groups, low expression of estrogen receptors was observed both in the glands and in the endometrial stroma. The expression of progesterone receptors in the glands and stroma of the endometrium was moderate. The determination of the stromal ratio of PR/Era expression revealed its increase: the control group to $3.44 \pm 0.69$; the experimental group to $3.72 \pm 0.82$ (at normal values from 2 to 3 units).

The immunological study performed after treatment revealed an increase in the absolute and relative content of $\mathrm{T}$ and $\mathrm{B}$ cells in patients of the experimental group ( $\mathrm{T}-$ lymphocytes $-69.2 \pm 2.4 \%$, $\mathrm{T}-$ helper cells $-83 \pm 1.4 \%$ ) $(\mathrm{p}<0.05)$, as well as a significant increase in interferonogenesis indicators (alpha-IFN up to $186 \pm 8.2 \%$, gamma-IFN - up to $73 \pm 10.2 \%)(\mathrm{p}<0.05)$. As a result of the stage-based rehabilitation, a significant decrease in pro-inflammatory indicators (IL-1, IL-6, TNF) and an 
increase in the concentration of anti-inflammatory cytokines (IL-4, IL-10) was revealed. The performed immunological studies confirmed the literature data on the influence of infectious and inflammatory processes on endometrial receptivity. The conducted complex etiotropic treatment helped to normalize the immune status of patients with chronic endometritis, and, consequently, their reproductive potential.

Positive changes in endometrial receptivity were characterized in the experimental group by the fact that none of the patients had glands with no expression to estrogens or progesterone. In the control group, only $10.0 \%$ of women maintained low expression to estrogens in the epithelium and the same number to progesterone. The dynamics of the studied parameters while investigating the ovarian hormone reception in the endometrial stroma in the experimental group showed that the number of patients with moderate or severe reception in the stroma to steroid hormones increased after pregravid preparation to $100 \%(\mathrm{p}<0.05)$. In the group receiving traditional treatment, the number of cases with low and zero expression of estrogens in the stroma decreased slightly. As for the expression of progesterone receptors, the changes in this category of patients were even less significant - low expression in the endometrial rhythm remained in $30.0 \%$ of patients.

Control histological examination of the endometrium after treatment, conducted in 78 patients with a confirmed diagnosis of chronic endometritis $(28$ - in the control group, 50 - in the experimental) demonstrated the restoration of the morphofunctional structure of the endometrium, respectively, in $18(41 \%)$ and $38(76 \%)$ of the examined patients. The endometrial biopsy results showed preserved cylindrical uterine endometrium with rare glandular tubes in a significant volume of loose stroma from process fibroblasts, the disappearance of inflammatory infiltrates, and a significant reduction in the area of sclerotic changes in the endometrial stroma in the experimental group in $84 \%$ of cases, in the control group - in $25 \%$. The comparative results of clinical and functional indicators of patients before and after treatment are presented in the table.

Table 1 The comparative results of clinical and functional indicators of patients $(M \pm m, p)$

\begin{tabular}{|l|c|c|c|c|c|c|}
\hline \multicolumn{1}{|c|}{ The features of changes } & \multicolumn{2}{|c|}{ Comparison group } & \multicolumn{2}{c|}{ Control group } & \multicolumn{2}{c|}{ Experimental group } \\
\cline { 2 - 6 } & $\begin{array}{c}\text { Before } \\
\text { treatment }\end{array}$ & $\begin{array}{c}\text { After } \\
\text { treatment }\end{array}$ & $\begin{array}{c}\text { Before } \\
\text { treatment }\end{array}$ & $\begin{array}{c}\text { After } \\
\text { treatment }\end{array}$ & $\begin{array}{c}\text { Before } \\
\text { treatment }\end{array}$ & $\begin{array}{c}\text { After } \\
\text { treatment }\end{array}$ \\
\hline $\begin{array}{l}\text { Pathology of menstrual } \\
\text { function \% }\end{array}$ & 82 & 72 & 92 & $42^{*}$ & 87 & $18^{* *}$ \\
\hline $\begin{array}{l}\text { Pathology of echo structures } \\
\text { of endometrium, \% }\end{array}$ & 100 & 81 & 100 & $48^{*}$ & 100 & $18^{* *}$ \\
\hline $\begin{array}{l}\text { Pathological hysteroscopic } \\
\text { condition of endometrium, } \%\end{array}$ & 57 & 40 & 63 & $10^{*}$ & 60 & 0 \\
\hline $\begin{array}{l}\text { Pathological morphological } \\
\text { condition of endometrium, } \%\end{array}$ & 100 & 73 & 100 & $27^{*}$ & 100 & $10^{* *}$ \\
\hline $\begin{array}{l}\text { Thickness M-ECHO in the - } \\
\text { post-ovulatory period }\end{array}$ & $6,24 \pm 0.72$ & $6,89 \pm 0.53$ & $6,71 \pm 0.7$ & $8,44 \pm 0,4^{*}$ & $6,58 \pm 0.76$ & $9,9 \pm 0,3^{* *}$ \\
\hline
\end{tabular}

Note: the reliability of differences in indicators is indicated in the table: $*-p<0,05 ; * *-p<0,01$.

Therefore, the performed complex etiotropic treatment with local intrauterine administration of drugs, so that they pass into the pelvic cavity, significantly increases the implantation potential of the endometrium by eliminating or reducing the manifestations of fibrosis, inflammation.

Improvement of reproductive potential and the onset of pregnancy are the most effective indicators of the effectiveness of complex etiotropic therapy in patients with chronic endometritis and infertility. Thus, within a year, $17 \%$ (5 out of 30 ) of the control group's patients became pregnant against the background of artificial insemination with their husband's sperm. In the experimental group, $30 \%$ (15 out of 50) of patients got pregnant during the 2 nd stage of treatment without assisted reproductive technologies, and $16 \%$ of patients got pregnant during artificial insemination at their condition. That is, in the experimental group, pregnancy occurred in $46 \%$ of patients with chronic endometritis and reproductive disorders, while in the control group - in 17 $\%$, which indicates the high effectiveness of the developed treatment regimen.

\section{CONCLUSION}

Thus, we have established the ability of intrauterine injection of drugs antibacterial, immunomodulatory and defibrotide action to recover macro - and microscopic, i.e. 
structural, but also the most important local gomeostaticeski, functional parameters of the endometrial expression of estrogen and progesterone receptors, which is a prerequisite for the proliferative and adequate secretory transformation of the correction of disorders of reproductive function and contributes to a comprehensive rehabilitation of the reproductive function of female patients with chronic endometritis.

\section{REFERENCES}

[1] E.P. Kellet, The role of the endometrium in failures of reproductive function, Probl. of reproduct. 2 (2010) 16-20.

[2] Yu.A. Lyzikova, Chronic endometritis as a cause of reproductive disorders in women, Probl. of health and ecology 3(45) (2015) 9-16, Gomel.

[3] I.A. Salov, D.T. Tashukhozhaeva, D.V. Marshalov, Yu.N. Tarasenko, The features of microcirculation and tissue oxygenation in patients with spontaneous abortion, Modern probl. of sci. and ed. 4 (2013) 124-126.

[4] K. Kitaya, T. Yasuo, Immunohistochemistrical and clinicopathological characterization of chronic endometritis, Amer. J. of Reproduct. Immunol. 66(5) (2011) 410-415.

[5] J.C. Kasius, H.M. Fatemi, C. Bourgain, The impact of chronic endometritis on reproductive outcome, Fertility and sterility 96(6) (2011) 1451-1456.

[6] O. Lebovitz, R. Orvieto, Treating patients with "thin" endometrium - an ongoing challenge, Gynecol. Endocrinol. 30(6) (2014) 409-414.

[7] L.V. Tsallagova, I.V. Kabulova et al., The role of chronic endometritis in the genesis of infertility, Kuban Sci. Med. Bull. 4 (2014) 131-136.

[8] J. Zolghadri, M. Momtahan, K. Aminian et al., The value of hysteroscopy in diagnosis of chronic endometritis in patients with unexplained recurrent spontaneous abortion, Eur. J. Obstet. Gynecol. Reprod. Biol. 155(2) (2011) 217-220. 